\title{
Hybrid density functional theory meets quasiparticle calculations: A consistent electronic structure approach
}

\author{
Viktor Atalla, ${ }^{1,2, *}$ Mina Yoon, ${ }^{2}$ Fabio Caruso, ${ }^{1}$ Patrick Rinke, ${ }^{1}$ and Matthias Scheffler ${ }^{1}$ \\ ${ }^{1}$ Fritz-Haber-Institut der Max-Planck-Gesellschaft, Faradayweg 4-6, 14195 Berlin, Germany \\ ${ }^{2}$ Center for Nanophase Materials Sciences, Oak Ridge National Laboratory, Oak Ridge, Tennessee 37831, USA
}

(Received 8 May 2012; revised manuscript received 11 June 2013; published 14 October 2013)

\begin{abstract}
We propose a scheme to obtain a system-dependent fraction of exact exchange $(\alpha)$ within the framework of hybrid density functional theory (DFT) that is consistent with the $G_{0} W_{0}$ approach, where $G_{0}$ is the noninteracting Green function of the system and $W_{0}$ the screened Coulomb interaction. We exploit the formally exact condition of exact DFT that the energy of the highest occupied molecular orbital corresponds to the ionization potential of a finite system. We identify the optimal $\alpha$ value for which this statement is obeyed as closely as possible and thereby remove the starting point dependence from the $G_{0} W_{0}$ method. This combined approach is essential for describing electron transfer (as exemplified by the TTF/TCNQ dimer) and yields the vertical ionization potentials of the $\mathrm{G} 2$ benchmark set with a mean absolute percentage error of only $\approx 3 \%$.
\end{abstract}

DOI: 10.1103/PhysRevB.88.165122

PACS number(s): 71.15.-m, 31.15.A-, 73.22.-f

\section{INTRODUCTION}

Predicting charge transfer from first principles is currently regarded as a great challenge., ${ }^{1,2}$ Kohn-Sham (KS) density functional theory (DFT) calculations with approximate functionals are plagued by the self-interaction error and the absence of the derivative discontinuity. As a consequence, artificial charge transfer between two molecules can result when the highest occupied molecular orbital (HOMO) level of one molecule erroneously moves above the lowest unoccupied molecular orbital (LUMO) level of the other. The solution would be to resort to a method that gives both correct (or at least improved) orbital energies and correct total energies.

One common way is to employ hybrid functionals that contain a fraction $\alpha$ of exact exchange within the generalized KS (GKS) approach. The problem translates into finding an appropriate $\alpha$ value. Theoretical considerations have produced global values of $1 / 4^{3,4}$ or $0.5,{ }^{5}$ neglecting the system dependence of $\alpha$, which, in principle arises from the inverse dependence on the dielectric function. ${ }^{6}$ In a pragmatic approach, $\alpha$ was rendered material dependent by fitting to experimental band gaps ${ }^{7}$ or to cohesive properties. ${ }^{8}$ However, fitting is theoretically unsatisfying, as it introduces an empirical parameter and it relies on the existence and accuracy of experimental data. For finite systems, an $a b$ initio way of determining $\alpha$ has recently been proposed ${ }^{9,10}$ by employing the difference in the self-consistent field ( $\triangle \mathrm{SCF})$ calculations approach.

An alternative solution to the level-alignment problem is found in many-body perturbation theory, e.g., the $G W$ approach. ${ }^{11}$ In order to obtain a well-defined total energy, $G W$ would have to be carried out fully self-consistently by solving the Dyson equation. This is computationally expensive and the subject of active research. ${ }^{12-14}$ Instead, the majority of all $G W$ calculations is carried out perturbatively, i.e., as a single-shot calculation taking wave function $\psi_{n \sigma}$ and orbital energy $\epsilon_{n \sigma}$ input from DFT (the so-called $G_{0} W_{0}$ approach):

$$
\epsilon_{n \sigma}^{G_{0} W_{0}}=\epsilon_{n \sigma}+\left\langle\psi_{n \sigma}\left|\Sigma^{G_{0} W_{0}}-v^{x c}\right| \psi_{n \sigma}\right\rangle .
$$

The indices $n$ and $\sigma$ label the main quantum numbers and spin states, respectively; $v^{x c}$ is the DFT exchange-correlation potential; and $\Sigma^{G_{0} W_{0}}$ is the $G W$ self-energy, which itself depends on $\psi_{n \sigma}$ and $\epsilon_{n \sigma}$. However, in $G_{0} W_{0}$ one looses access to the ground-state properties and introduces a starting-point dependence. ${ }^{15-20}$ For charge transfer between two molecules this implies that even if one were to apply $G_{0} W_{0}$ for a given DFT functional, one could not be sure that it would give sensible results. Let us assume that we start with a DFT functional that gives erroneous charge transfer. The subsequent $G_{0} W_{0}$ calculation is now based on an incorrect density. Moreover, even if $G_{0} W_{0}$ were to produce a level alignment that does not give rise to charge transfer, we could not utilize this result, because $G_{0} W_{0}$ does not give us a new density, a new total energy, or new wave functions. In other words, there is no way back from the $G_{0} W_{0}$ spectrum to the ground-state properties of the system. In this paper we propose a scheme that solves this problem, by making the DFT starting point internally consistent with $G_{0} W_{0}$ and, thereby, lifting the ambiguity in the choice of $\alpha$.

\section{THEORY}

We consider the Perdew-Burke-Ernzerhof (PBE) hybrid functional (PBEh) $)^{3,21}$ and make the XC energy $E_{\mathrm{xc}}$ explicitly $\alpha$ dependent:

$$
E_{\mathrm{xc}}=\alpha E_{\mathrm{x}}^{\mathrm{EX}}+(1-\alpha) E_{\mathrm{x}}^{\mathrm{PBE}}+E_{\mathrm{c}}^{\mathrm{PBE}}, \quad \alpha \in[0,1] .
$$

Here $E_{\mathrm{x}}^{\mathrm{PBE}}$ and $E_{\mathrm{c}}^{\mathrm{PBE}}$ denote the PBE exchange and correlation energy, respectively, ${ }^{21}$ and $E_{\mathrm{x}}^{\mathrm{EX}}$ is the exact exchange energy. In order to emphasize the $\alpha$ dependence in the exchange part we introduce the notation $\operatorname{PBEh}(\alpha)$ for the corresponding functional. For example, $\alpha=0$ corresponds to the $\mathrm{PBE}^{21}$ and $\alpha=0.25$ to the PBE $0^{3}$ functional. Instead of fixing $\alpha$ globally, we consider it a system-dependent parameter (which, in principle, depends on the electron density by virtue of the Hohenberg-Kohn theorem). ${ }^{22}$ For a given system we then choose $\alpha$ by minimizing the quasiparticle (QP) correction to 
the HOMO level according to Eq. (1),

$$
\alpha^{*}=\underset{\alpha}{\arg \min }\left|\left\langle\psi_{\mathrm{H}}(\alpha)\left|\Sigma(\alpha)-v^{x c}(\alpha)\right| \psi_{\mathrm{H}}(\alpha)\right\rangle\right|,
$$

where the index $\mathrm{H}$ denotes the HOMO level. The $\alpha$ dependence of the XC potential, the KS orbitals, and the self-energy has been taken into account explicitly. In exact DFT the HOMO level of a finite system can be rigorously assigned to the ionization potential (IP), ${ }^{23,24}$ and therefore the self-energy correction to the HOMO is strictly 0 . No such statement holds for any other KS states. However, even the HOMO is typically not given accurately in standard approximations to the XC functional, because of the self-interaction error (SIE). ${ }^{25}$ Equation (3) therefore requires that the self-energy correction to the HOMO level is as small as possible. This is equivalent to demanding that the correspondence between the HOMO and the IP in exact DFT is obeyed as closely as possible. Hence, from a DFT perspective Eq. (3) also reduces the self-interaction error. Since the exact self-energy $\Sigma$ in Eq. (3) is not easier to calculate than the exact XC potential, we approximate it by the $G W$ self-energy $\Sigma^{G_{0} W_{0}}$. Equation (3) can then also be viewed as the definition of an internally consistent DFT starting point to $G_{0} W_{0}$.

In practice, Eq. (3) can be solved by just a few singleshot $G_{0} W_{0}$ calculations, which makes it computationally much more efficient than fully self-consistent $G W$ calculations. $\alpha^{*}$ can be obtained by interpolation to that $\alpha$ value, for which the difference between the KS and the QP HOMO level is minimized. For almost all systems we have considered (for example, see Table V), we found $\alpha^{*}$ values for which the KS and the QP HOMO levels can be matched implying a vanishing QP correction.

Our scheme is conceptually similar to the QP self-consistent $G W(\mathrm{QS} G W)$ theory proposed by Schilfgaarde and coworkers. ${ }^{26}$ However, in our scheme the choice of the optimum potential is restricted to a subset that emerges from hybrid KS DFT. As a consequence, the corresponding ground-state energy is still variational with respect to the density, unlike in QSGW. We also note a recent paper by Körzdörfer and Marom, ${ }^{18}$ who suggest an optimal, consistent $G_{0} W_{0}$ starting point that obtains $\alpha$ by a linear regression over the full occupied spectrum. In contrast, we obtain $\alpha$ by requiring the fulfillment of only the HOMO IP condition [Eq. (3)], which is exact for the exact XC functional and the exact single-particle Green's function and approximately exact for the PBEh family and the $G_{0} W_{0}$ method, respectively. A comparison between this and our method is given in this paper for the TTF/TCNQ dimer (Sec. III C).

\section{THE TTF/TCNQ DIMER}

\section{A. The individual molecules}

We apply our scheme to the TTF/TCNQ dimer, a prototypical donor/acceptor system in the field of organic electronics. ${ }^{27-29}$ For this dimer, it was recently shown that standard local, semilocal, and hybrid XC functionals can fail drastically. ${ }^{2}$ First, we consider the individual molecules. We adopt the notation "method@functional"; for example, $G_{0} W_{0} @ \mathrm{PBE}$ corresponds to a $G_{0} W_{0}$ calculation with PBE reference states. Figures 1(a) and 1(b) show the KS and

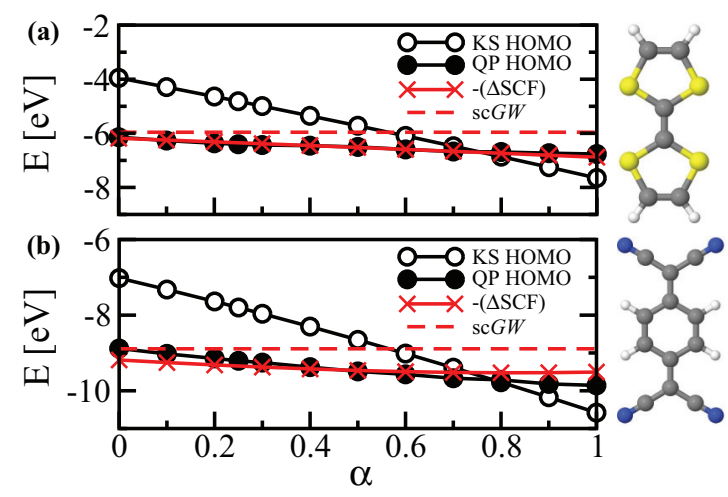

FIG. 1. (Color online) QP (filled circles), KS (open circles), $\triangle \mathrm{SCF}[(\mathrm{red})$ crosses], and $\operatorname{sc} G W$ [dashed (red) line] HOMO level as a function of $\alpha$ for the (a) TTF and (b) TCNQmolecules. The molecules are shown at the right: White denotes hydrogen; gray, carbon; blue, nitrogen; yellow, sulfur.

QP HOMO levels as a function of $\alpha$. The calculations were performed using the all-electron, numeric atom-centered orbital code FHI-aims. ${ }^{30,31}$ The geometry of the individual molecules was optimized in PBE with a tier 2 basis. All $G_{0} W_{0}$ calculations were performed with a tier 3 basis. We obtain $\alpha^{*}=0.8$ for both TTF and TCNQ. Similar values for $\alpha$ were reported ${ }^{32}$ for benzene $(\alpha=0.7)$, bithiophene $(\alpha=0.6)$, and benzoquinone $(\alpha=0.5)$ by minimizing the many-electron self-interaction error. ${ }^{33}$ These are all much higher than the $\alpha=0.25$ used in the PBE0 functional.

We compared our method with the negative value of the $\triangle \mathrm{SCF}$ approach in Ref. 9. Figures 1(a) and 1(b) show that the $\triangle$ SCF IPs lie essentially on top of the $G_{0} W_{0}$ line. This indicates that for finite systems (for which the $\triangle \mathrm{SCF}$ approach usually yields good results) both methods agree closely.

Experimentally, the IP of TTF obtained by photoelectron spectroscopy is $6.70 \mathrm{eV}^{34}$ The IPs for the different $G_{0} W_{0}$ starting points are sumarized in Table I, where the IP is obtained from the negative HOMO value. $G_{0} W_{0} @ \mathrm{PBE}$ and $G_{0} W_{0} @$ PBE0 underestimate the IP by $9.1 \%$ and $4.7 \%$, whereas $G_{0} W_{0} @ \operatorname{PBEh}\left(\alpha^{*}\right)$ slightly overestimates it, by $0.6 \%$. TCNQ has an experimental IP of $9.61 \mathrm{eV}^{35}$ In comparison, the calculated $G_{0} W_{0}$ IPs underestimate this value by $8.2 \%$ (4.3\%) for the PBE (PBE0) starting point and overestimate it by $1.0 \%$ for the $\operatorname{PBEh}\left(\alpha^{*}\right)$ starting point. In both cases, the IPs

TABLE I. Ionization potential (IP) for TTF and IP and electron affinity (EA) for TCNQ obtained with $G_{0} W_{0}$ for various starting points using the tier 3 basis set. Experimental IPs and the EA of TCNQ obtained with $\operatorname{CCSD}(\mathrm{T})$ are also listed.

\begin{tabular}{lccc}
\hline \hline & \multirow{2}{*}{ TTF } & \multicolumn{2}{c}{ TCNQ } \\
\cline { 3 - 4 } & IP & IP & EA \\
\hline$G_{0} W_{0} @$ PBE & 6.14 & 8.88 & 4.06 \\
$G_{0} W_{0} @$ PBE0 & 6.40 & 9.21 & 3.95 \\
$G_{0} W_{0} @$ PBEh $\left(\alpha^{*}\right)$ & 6.66 & 9.70 & 3.73 \\
Expt. & $6.7^{34}$ & $9.61^{35}$ & - \\
CCSD $(\mathrm{T})$ & - & - & $3.22^{36}$ \\
\hline \hline
\end{tabular}


obtained by $G_{0} W_{0} @ \operatorname{PBEh}\left(\alpha^{*}\right)$ are in excellent agreement with the experimental values; otherwise, they are underestimated.

Figures 1(a) and 1(b) also show fully self-consistent $G W$ $(\operatorname{sc} G W)$ results for the IPs, which appear as constant lines since $\operatorname{sc} G W$ is independent of the starting point. ${ }^{12}$ For TTF and TCNQ, sc $G W$ underestimates the IP more than for other closed-shell molecules (see, e.g., Refs. 12,19, and Table VI in the Appendix). This would reduce the optimal $\alpha$ to $\sim 0.6$. The reason for the large errors in $\operatorname{sc} G W$ for TTF and TCNQ is not clear at present and will be the subject of future research.

Coupled cluster calculations including single, double, and perturbative triple excitations $[\mathrm{CCSD}(\mathrm{T})]$ predict an electron affinity (EA) of $3.22 \mathrm{eV}^{36}$ for TCNQ. The TCNQ EAs for the different $G_{0} W_{0}$ starting points are also sumarized in Table I. Compared to $\mathrm{CCSD}(\mathrm{T})$, the TCNQ EAs are overestimated by $26.1 \%$ for $G_{0} W_{0} @ \mathrm{PBE}, 22.7 \%$ for $G_{0} W_{0} @ \mathrm{PBE} 0$, and $15.8 \%$ for $G_{0} W_{0} @ \operatorname{PBEh}\left(\alpha^{*}\right)$. Hence, all $G_{0} W_{0}$ results give EAs that are too high compared to $\operatorname{CCSD}(\mathrm{T})$ results. However, the lowest error is obtained for $G_{0} W_{0} @ \operatorname{PBEh}\left(\alpha^{*}\right)$, even though the LUMO level is not explicitly taken into account in Eq. (3).

\section{B. The dimer}

Next, we evaluate the impact of $\alpha$ on dipole moments and electron transfer for the TTF/TCNQ dimer. The geometry of the dimer (Fig. 2, right) has been cut out of a TTF/TCNQ interface along the [001] surface of TCNQ. ${ }^{37}$ Figure 2(a) shows the absolute values of the dipole moment as a function of distance. At the interface equilibrium distance $\left(d_{0}\right)$ the dipole moment differs significantly among PBE, PBE0, and $\operatorname{PBEh}\left(\alpha^{*}\right)$. More importantly, however, the dipole moment for PBE and PBE0 does not vanish at long distances. Experimentally, both molecules have a zero dipole moment in the gas phase. The dipole moment of the dimer in the long-range limit should therefore also be 0 , because at infinite separation the total dipole moment is given by the sum of the two individual dipole moments. Only the $\operatorname{PBEh}\left(\alpha^{*}\right)$ functional reproduces the correct asymptotic limit. The unphysical behavior of PBE and PBE0 in the long-range limit can be traced back to their erroneous description of electron transfer, as also shown in Fig. 2(b). Both PBE and PBE0 predict a nonvanishing electron

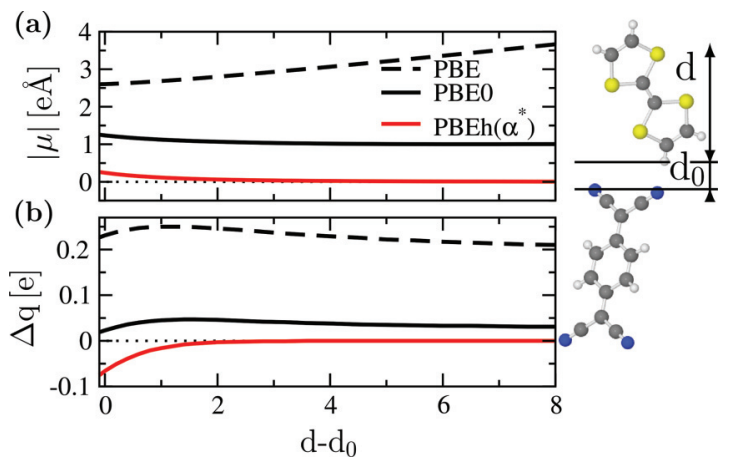

FIG. 2. (Color online) Absolute value of the dipole moment (a) and electron transfer (b) for the TTF/TCNQ complex as a function of the dimer distance. A positive sign indicates electron transfer from TTF to TCNQ.

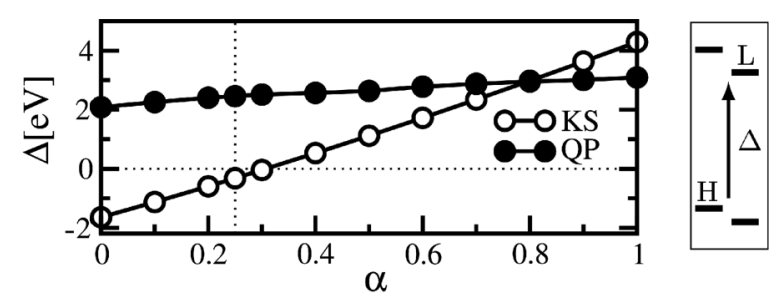

FIG. 3. Differences $(\Delta)$ in the generalized KS LUMO (L) of TCNQ vs HOMO $(\mathrm{H})$ of TTF as a function of $\alpha$ (open circles) and corresponding $G_{0} W_{0}$ values (filled circles). A negative $\Delta$ value indicates artificial electron transfer in the noninteracting limit. The dotted vertical line corresponds to the PBE0 result.

transfer in the long-range limit, which subsequently induces a nonvanishing dipole moment.

To proceed, we consider the limit of infinite molecular separation. In this limit, electron transfer between the molecules is determined by the difference between the EA of TCNQ and the IP of TTF. Since the CCSD(T) and $G_{0} W_{0}$ EA of TCNQ is smaller than the IP of TTF, there should be no electron transfer at infinite separation. This indicates that both PBE and PBE0 fail to describe the ground-state density of the TTF/TCNQ dimer. Within the framework of KS DFT the total electron density is built up by occupying orbitals with respect to their energetic ordering. It is thus the relative alignment of the TCNQ LUMO and the TTF HOMO that determines electron transfer between the two molecules in this limit. Figure 3 illustrates the difference between the GKS LUMO of TCNQ and the HOMO of TTF, $\Delta$, as a function of $\alpha$. For $\alpha<0.3$ the TCNQ LUMO level is located below the TTF HOMO level. Thus, TCNQ will receive a certain amount of charge just by occupying orbitals with respect to their energetic ordering. For $\alpha>0.3$ the level alignment of the TTFTCNQ frontier orbitals qualitatively agrees with experiment and $\operatorname{CCSD}(\mathrm{T})$ for which $\Delta$ is positive. ${ }^{2}$ In particular, both $\operatorname{PBE}(\alpha=0)$ and PBE0 $(\alpha=0.25)$ are below the critical $\alpha$ value of 0.3 and therefore have an artificial contribution to electron transfer. $\operatorname{PBEh}\left(\alpha^{*}\right)$, on the other hand, gives a level alignment that is consistent with experiment $[\mathrm{CCSD}(\mathrm{T})]$ and a vanishing QP correction to $\Delta$. As a consequence, the tuned PBEh functional is free of spurious asymptotic electron transfer.

\section{Comparison to the consistent $G_{0} W_{0}$ starting-point scheme}

We now turn to a comparison between our method and the consistent $G_{0} W_{0}$ starting point scheme (CSP) suggested by Körzdörfer and Marom. ${ }^{18}$ The starting point of the CSP scheme is to separate the linearized QP equation for the $n$th QP energy (within the $G_{0} W_{0}$ approximation) into its exchange and correlation parts:

$$
\begin{aligned}
\epsilon_{n}^{G_{0} W_{0}}= & \epsilon_{n}+\left\langle\psi_{n},\left(\Sigma^{G_{0} W_{0}}-v^{x c}\right) \psi_{n}\right\rangle \\
= & \epsilon_{n}+\left\langle\psi_{n},\left(\Sigma_{c}^{G_{0} W_{0}}-v^{c}\right) \psi_{n}\right\rangle \\
& +(1-\alpha)\left\langle\psi_{n},\left(v_{x}^{\mathrm{EX}}-v_{x}^{\mathrm{PBE}}\right) \psi_{n}\right\rangle \\
= & : \epsilon_{n}+\Delta v_{c, n}+(1-\alpha) \Delta v_{x, n},
\end{aligned}
$$

where $v_{x}^{\mathrm{EX}}$ and $v_{x}^{\mathrm{PBE}}$ are the exact-exchange and PBE-exchange potentials, respectively. Körzdörfer and Marom require the 
TABLE II. Ionization potential (IP) and electron affinity (EA; eV) for the TTF and TCNQ molecules obtained by taking the negative of the $G_{0} W_{0}$ HOMO and LUMO level (in parentheses are the corresponding GKS values). The bottom two rows summarize the experimental IPs and the TCNQ EA obtained by $\operatorname{CCSD}(\mathrm{T})$.

\begin{tabular}{lccccc}
\hline \hline & \multicolumn{3}{c}{ TTF } & & \multicolumn{2}{c}{ TCNQ } \\
\cline { 2 - 3 } \cline { 5 - 6 } \cline { 5 - 6 } & IP & EA & & IP & EA \\
\hline $\operatorname{PBEh}\left(\alpha^{*}\right)$ & $6.66(6.66)$ & $-0.67(-0.68)$ & & $9.70(9.70)$ & $3.73(3.91)$ \\
$\operatorname{PBEh}(\bar{\alpha})$ & $6.27(4.29)$ & $-0.17(1.61)$ & & $9.19(7.76)$ & $3.95(5.15)$ \\
$\operatorname{Exp}$. & $6.7^{34}$ & - & & $9.61^{35}$ & - \\
$\operatorname{CCSD}(\mathrm{T})$ & - & - & & - & $3.22^{36}$ \\
\hline \hline
\end{tabular}

relative $G_{0} W_{0}$ shift of the occupied eigenvalues $\left\{\epsilon_{n}, n \in\right.$ Occ $\}$ to be as small as possible:

$$
\Delta v_{c, n}+(1-\alpha) \Delta v_{x, n} \approx \text { const, } \quad n \in \text { Occ. }
$$

Satisfaction of the requirement of Eq. (5) yields the optimal value of $\alpha$ (denoted $\bar{\alpha}$ ) as follows: For a given guess of $\alpha$ the self-consistent $(\mathrm{G}) \mathrm{KS}$ eigenvalues and orbitals are calculated. These eigenvalues are used to calculate $\Delta v_{c, n}$ and $\Delta v_{x, n}$ according to Eq. (4). Then a straight line is fit to the $\Delta v_{c, n}$ vs $\Delta v_{x, n}$ dependence. This determines a new $\alpha$, which is used to continue the iteration. When the slope determined by the linear fit equals the slope in Eq. (5), $\bar{\alpha}$ has been found. By construction, $\operatorname{PBEh}(\bar{\alpha})$-i.e., a PBEh functional with a fraction $\bar{\alpha}$ of exact exchange-represents an optimum starting point for $G_{0} W_{0}$ calculations of the occupied spectrum. For TTF Körzdörfer and Marom obtain $\bar{\alpha}=0.1$, and for TCNQ $\bar{\alpha}=0.24 .^{18}$ Both values are much smaller than in our scheme and are also below the value of 0.3 required for interaction-free charge transfer.

Table II compares the EA and the IP of $\operatorname{PBEh}(\bar{\alpha})$ vs $\operatorname{PBEh}\left(\alpha^{*}\right)$. On the $G_{0} W_{0}$ side, the $\operatorname{PBEh}(\bar{\alpha})$-based IPs tend to be underestimated with respect to both experiment and $\operatorname{PBEh}\left(\alpha^{*}\right)$. The EAs, on the other hand, are overestimated compared to $\operatorname{PBEh}\left(\alpha^{*}\right)$ and $\operatorname{CCSD}(\mathrm{T})$. On the GKS level of theory, this trend is significantly enhanced. In particular, $\operatorname{PBEh}(\bar{\alpha})$ moves the IP of TTF incorrectly above the EA of TCNQ and hence predicts erroneous interaction-free electron transfer from TTF to TCNQ.

Körzdörfer and Marom argue that any scheme that is optimized only on the frontier orbitals of the system may not provide good spectral properties for the whole excitation spectrum, ${ }^{38}$ unlike the CSP scheme they propose. ${ }^{18}$ The CSP scheme, on the other hand, fails for the ground state of the TTF/TCNQ dimer. A challenge for future developments

TABLE III. Mean absolute percentage error (MAPE) of theoretical IPs obtained from $G_{0} W_{0}$, (generalized) KS HOMO levels, and $\triangle \mathrm{SCF}$ with respect to experimental vertial IPs for 50 molecules in the $\mathrm{G} 2$ test set.

\begin{tabular}{lrrc}
\hline \hline & PBE & PBE0 & $\operatorname{PBEh}\left(\alpha^{*}\right)$ \\
\hline$G_{0} W_{0} @$ & 4.5 & 2.2 & 2.9 \\
HOMO & 39.4 & 27.0 & 2.9 \\
$\triangle \mathrm{SCF}$ & 2.2 & 2.1 & 3.1 \\
\hline \hline
\end{tabular}

TABLE IV. Mean absolute error (MAE; in meV) of different total energy methods for the $\mathrm{S} 22$ database $^{41}$ with respect to $\operatorname{CCSD}(\mathrm{T})$ results. $^{42}$

\begin{tabular}{lcccr}
\hline \hline & H bonds & Dispersion & Mixed & Total \\
\hline $\operatorname{PBE}$ & 51 & 198 & 87 & 116 \\
$\operatorname{PBEh}\left(\alpha^{*}\right)$ & 36 & 164 & 55 & 89 \\
$(\mathrm{EX}+\mathrm{cRPA}) @ \operatorname{PBE}$ & 55 & 34 & 24 & 37 \\
$(\mathrm{EX}+\mathrm{cRPA}) @ \operatorname{PBEh}\left(\alpha^{*}\right)$ & 21 & 54 & 24 & 34 \\
\hline \hline
\end{tabular}

is therefore to devise schemes that give good ground- and excited-state properties.

\section{THE G2 BENCHMARK SET}

We also examined the performance of our scheme for a subset of the G2 test set for ionization energies, ${ }^{39}$ consisting of 50 atoms and molecules for which experimental geometries and vertical IPs are available. ${ }^{40}$ All calculations were carried out for experimental geometries using the tier 4 FHI-aims basis $^{30}$ augmented with diffuse functions from the aug-ccpV5Z. Following Eq. (3) we obtain $\alpha^{*}$ values $\geqslant 0.7$ for all the atoms and molecules in the subset. The detailed numerical results are compiled in Table $\mathrm{V}$ in the Appendix. Table III summarizes the results in comparison to the experimental reference data for the $G_{0} W_{0}$ and $(\mathrm{G}) \mathrm{KS}$ IPs, which were obtained by taking the negative of the respective HOMO level as well as by the $\triangle \mathrm{SCF}$ approach.

For the given test set, the dependence of $G_{0} W_{0}$ on the fraction of exact exchange was found to be relatively weak. $G_{0} W_{0}$ based on PBE reference states performs very well even though it tends to underestimate the IP and has a mean absolute percentage error (MAPE) of 4.5\%. $G_{0} W_{0} @ \mathrm{PBE} 0$ and $G_{0} W_{0} @ \operatorname{PBEh}\left(\alpha^{*}\right)$ perform similarly, with a MAPE of $2.2 \%$ and $2.9 \%$, respectively, and thus further reduce the error compared to $G_{0} W_{0} @ \mathrm{PBE}$. In contrast to $G_{0} W_{0}$, the KS HOMO depends quite sensitively on $\alpha$. In particular, PBE underestimates the IP by as much as $39.4 \%$. An $\alpha$ value of $1 / 4$ as used in PBE0 improves the IPs over PBE, however, the error is still as large as $27.0 \%$. A realistic description can only be achieved by $\operatorname{PBEh}\left(\alpha^{*}\right)$, which brings the MAPE down to $2.9 \%$.

The $\triangle \mathrm{SCF}$ method gives small errors, which are similar to those of $G_{0} W_{0}$ for all three functionals. For $\operatorname{PBEh}\left(\alpha^{*}\right)$ this implies (i) that it is compatible with the $\triangle \mathrm{SCF}$ method for the calculation of IPs and (ii) that the $\triangle \mathrm{SCF}$ approach for the determination of $\alpha^{9}$ is applicable for the PBE hybrid family of $\mathrm{XC}$ functionals.

\section{THE S22 BENCHMARK SET}

We also tested the performance of the $\operatorname{PBEh}\left(\alpha^{*}\right)$ functional for binding energies in the $S 22^{41}$ data set, which contains hydrogen, dispersion-dominated, and mixed types of bonds. Table IV summarizes the results compared to $\operatorname{CCSD}(\mathrm{T})$ reference values. ${ }^{42}$ On the DFT level of theory we find that $\operatorname{PBEh}\left(\alpha^{*}\right)$ improves the binding energies with respect to $\mathrm{PBE}$ 
and PBE0 (not shown) for all types of bonding present in the S22 data set. However, in particular, dispersion-dominated systems come out poorly since the PBEh family of XC functionals does not include long-range van der Waals interactions. These interactions can be added in the exact exchange plus correlation in the random phase approximation (cRPA) ${ }^{43}$ framework. We find that $(\mathrm{EX}+\mathrm{cRPA}) @ \operatorname{PBEh}\left(\alpha^{*}\right)$ performs better for $\mathrm{H}$ bonds (MAE, $21 \mathrm{meV}$ ) than (EX + cRPA)@PBE (MAE, $55 \mathrm{meV}$ ), whereas this trend is reversed for dispersion interactions. However, the overall performance of EX + cRPA is very similar for both PBE- and $\operatorname{PBEh}\left(\alpha^{*}\right)$-based reference states, which have a MAE of 37 and $34 \mathrm{meV}$, respectively. Thus we conclude that our method is compatible with RPA. We would like to note that more sophisticated RPA-based approaches exist, e.g., including renormalized second-order perturbation theory (r2PT), ${ }^{44,45}$ which brings the total MAE down to $21 \mathrm{meV}$.

\section{CONCLUSIONS}

Finally, we note that our proposed scheme relies on the accuracy of the $G_{0} W_{0}$ approximation to the single-particle Green's function itself. In particular, vertex corrections or a residual self-interaction error present in the $G_{0} W_{0}$ selfenergy $^{46}$ may affect the scheme for certain systems. Furthermore, for combined systems, where each subsystem requires different $\alpha^{*}$ values, e.g., molecules on surfaces, it is not clear whether a single choice of $\alpha$ may give a satisfactory description for the whole system. We plan to investigate these issues further in the future.

In conclusion, we have presented a scheme that obtains a system-dependent fraction of exact exchange by combining hybrid DFT and the $G_{0} W_{0}$ method. We obtain a hybrid functional that is both consistent in the choice of the fraction of exact exchange and consistent as a $G_{0} W_{0}$ starting point. The former implies an improvement of the generalized KS spectrum and thus an improved electron density.

\section{ACKNOWLEDGMENTS}

V.A. acknowledges partial support provided by a Laboratory Directed Research and Development award from Oak Ridge National Laboratory (ORNL). M.Y. was supported by the Scientific User Facilities Division at the Center for Nanophase Materials Sciences at ORNL by the Office of Basic Energy Sciences, US Department of Energy, and National Energy Research Scientific Computing Center under Contract No. DE-AC02-05CH11231.

\section{APPENDIX: NUMERICAL RESULTS FOR THE G2 TEST SET}

In the following, the results for a subset of 50 atoms and molecules from the $\mathrm{G} 2$ test set $^{39}$ of ionization energies are presented. The reference experimental IPs were taken from the NIST database. ${ }^{40}$ Calculations were performed using tier 4 basis sets augmented with diffuse functions from aug-cc-pV5Z.

The fraction of exact exchange $\left(\alpha^{*}\right)$ was determined by first performing single-point calculations $G_{0} W_{0} @ \operatorname{PBEh}(\alpha)$ for $\alpha=0.0,0.25,0.5,0.75,1.0$, followed by a quadratic fit for the difference in the KS vs QP HOMO energies. The minimum of the quadratic fit function is then determined analytically on the compact interval $[0,1]$. The set of computed $\alpha$ values gives QP and GKS HOMO levels that agree within $0.1 \mathrm{eV}$ at $\alpha^{*}$. A higher accuracy can be obtained by computing more points near the optimum $\alpha$. Apart from four exceptions (Be, $\mathrm{Mg}, \mathrm{N}$, and $\mathrm{NaCl}), \alpha$ could be determined such that the QP correction not only is minimized but vanishes, implying a KS HOMO level that energetically coincides with the corresponding $G_{0} W_{0}$ value. For the exceptions we found that $\alpha^{*}=1.0$ and that the KS HOMO level deviates less than $0.2 \mathrm{eV}$ with respect to the corresponding $G_{0} W_{0}$ HOMO.

Table V summarizes the $\alpha^{*}$ values obtained in this way and the corresponding negative of the KS and QP HOMO energies. For comparison also the corresponding $G_{0} W_{0} @ \mathrm{PBE}, G_{0} W_{0} @ \mathrm{PBE} 0$, and $\Delta \mathrm{SCF}$ for $\operatorname{PBEh}\left(\alpha^{*}\right)$ results (using the tier 4 basis augmented with diffuse functions from aug-cc-pV5Z) as well as the experimental values ${ }^{40}$ are tabulated.

Table VI compares the $\operatorname{sc} G W$ and $G_{0} W_{0} @ \operatorname{PBEh}\left(\alpha^{*}\right)$ IPs for a subset of closed-shell molecules in the G2 test set. ${ }^{39}$ For comparison, the $\operatorname{sc} G W$ results are shown for the tier 2 basis set, whereas the $G_{0} W_{0}$ results are tabulated for the tier 4 basis augmented with diffuse functions from aug-cc-pV5Z. Also tabulated are experimental reference values. $^{40}$

TABLE V. Optimized $\alpha^{*}$ values and ionization potentials $(\mathrm{eV})$ obtained by taking the negative of the $\operatorname{PBEh}\left(\alpha^{*}\right)$ and $G_{0} W_{0} @ \operatorname{PBEh}\left(\alpha^{*}\right)$ HOMO level for 50 molecules in the G2 test set. Also listed are the experimental (Expt.), $G_{0} W_{0} @ \mathrm{PBE}, G_{0} W_{0} @ \mathrm{PBE} 0$, and $\Delta \mathrm{SCF}$ for PBEh( $\left.\alpha^{*}\right)$ values. The mean absolute error (MAE) and mean absolute percentage error (MAPE) are summarized in the last two rows.

\begin{tabular}{|c|c|c|c|c|c|c|c|}
\hline Molecule & Expt. & $\alpha^{*}$ & $\begin{array}{c}\text { GKS } \\
\operatorname{PBEh}\left(\alpha^{*}\right)\end{array}$ & $G_{0} W_{0} @ \operatorname{PBEh}\left(\alpha^{*}\right)$ & $\begin{array}{c}\Delta \mathrm{SCF} \\
\operatorname{PBEh}\left(\alpha^{*}\right)\end{array}$ & $G_{0} W_{0} @ \mathrm{PBE}$ & $G_{0} W_{0} @ \mathrm{PBE} 0$ \\
\hline $\mathrm{Al}$ & 5.99 & 0.92 & 6.20 & 6.18 & 6.18 & 5.64 & 5.94 \\
\hline $\mathrm{Ar}$ & 15.76 & 0.82 & 15.90 & 15.89 & 15.73 & 15.21 & 15.51 \\
\hline B & 8.30 & 0.90 & 8.63 & 8.60 & 8.61 & 7.65 & 8.11 \\
\hline $\mathrm{BCl}_{3}$ & 11.64 & 0.76 & 12.11 & 12.08 & 12.35 & 11.24 & 11.62 \\
\hline $\mathrm{BF}_{3}$ & 15.96 & 0.70 & 16.34 & 16.38 & 16.70 & 15.19 & 15.79 \\
\hline $\mathrm{Be}$ & 9.32 & 1.00 & 9.13 & 9.18 & 8.90 & 9.26 & 9.10 \\
\hline $\mathrm{C}$ & 11.26 & 0.86 & 11.58 & 11.55 & 11.48 & 10.47 & 10.93 \\
\hline
\end{tabular}


TABLE V. (Continued.)

\begin{tabular}{|c|c|c|c|c|c|c|c|}
\hline Molecule & Expt. & $\alpha^{*}$ & $\begin{array}{c}\text { GKS } \\
\operatorname{PBEh}\left(\alpha^{*}\right)\end{array}$ & $G_{0} W_{0} @ \operatorname{PBEh}\left(\alpha^{*}\right)$ & $\begin{array}{c}\Delta \mathrm{SCF} \\
\operatorname{PBEh}\left(\alpha^{*}\right)\end{array}$ & $G_{0} W_{0} @ \mathrm{PBE}$ & $G_{0} W_{0} @ \mathrm{PBE} 0$ \\
\hline $\mathrm{C}_{2} \mathrm{H}_{2}$ & 11.49 & 0.88 & 11.59 & 11.62 & 10.95 & 11.01 & 11.30 \\
\hline $\mathrm{C}_{2} \mathrm{H}_{4}$ & 10.68 & 0.88 & 10.70 & 10.71 & 10.04 & 10.20 & 10.44 \\
\hline $\mathrm{C}_{2} \mathrm{H}_{4} \mathrm{~S}$ thiirane & 9.05 & 0.79 & 9.31 & 9.31 & 8.94 & 8.73 & 8.98 \\
\hline $\mathrm{C}_{2} \mathrm{H}_{5} \mathrm{OH}$ & 10.64 & 0.75 & 11.23 & 11.20 & 10.55 & 10.21 & 10.66 \\
\hline $\mathrm{C}_{6} \mathrm{H}_{6}$ & 9.25 & 0.80 & 9.42 & 9.40 & 9.10 & 9.01 & 9.24 \\
\hline $\mathrm{CH}_{2} \mathrm{CCH}_{2}$ & 10.20 & 0.84 & 10.53 & 10.55 & 9.93 & 9.84 & 10.18 \\
\hline $\mathrm{CH}_{2} \mathrm{~S}$ & 9.38 & 0.81 & 9.56 & 9.54 & 9.20 & 9.01 & 9.26 \\
\hline $\mathrm{CH}_{3}$ & 9.84 & 0.80 & 10.01 & 10.03 & 9.86 & 9.26 & 9.60 \\
\hline $\mathrm{CH}_{3} \mathrm{Cl}$ & 11.29 & 0.80 & 11.68 & 11.66 & 11.38 & 11.02 & 11.30 \\
\hline $\mathrm{CH}_{3} \mathrm{~F}$ & 13.04 & 0.78 & 13.79 & 13.78 & 13.50 & 12.85 & 13.27 \\
\hline $\mathrm{CH}_{3} \mathrm{SH}$ & 9.44 & 0.81 & 9.63 & 9.65 & 9.32 & 9.06 & 9.30 \\
\hline $\mathrm{CH}_{4}$ & 13.60 & 0.84 & 14.71 & 14.70 & 14.29 & 13.99 & 14.30 \\
\hline $\mathrm{CHO}$ & 9.31 & 0.78 & 10.33 & 10.26 & 10.55 & 9.14 & 9.63 \\
\hline $\mathrm{CO}$ & 14.01 & 0.81 & 14.65 & 14.56 & 14.25 & 13.31 & 13.83 \\
\hline $\mathrm{CO}_{2}$ & 13.78 & 0.76 & 14.22 & 14.18 & 13.75 & 13.16 & 13.66 \\
\hline $\mathrm{CS}_{2}$ & 10.09 & 0.82 & 10.34 & 10.30 & 9.96 & 9.71 & 9.95 \\
\hline $\mathrm{Cl}$ & 12.97 & 0.84 & 13.15 & 13.12 & 12.94 & 12.62 & 12.83 \\
\hline $\mathrm{Cl}_{2}$ & 11.49 & 0.80 & 11.91 & 11.84 & 11.98 & 11.18 & 11.50 \\
\hline $\mathrm{ClF}$ & 12.77 & 0.82 & 11.49 & 11.50 & 11.36 & 10.84 & 11.12 \\
\hline $\mathrm{F}$ & 17.42 & 0.78 & 17.51 & 17.50 & 17.05 & 16.71 & 17.07 \\
\hline FH & 16.12 & 0.74 & 16.29 & 16.29 & 15.69 & 15.39 & 15.83 \\
\hline $\mathrm{H}$ & 13.60 & 0.96 & 13.61 & 13.62 & 13.76 & 12.65 & 13.08 \\
\hline $\mathrm{He}$ & 24.59 & 0.89 & 24.65 & 24.63 & 24.43 & 23.59 & 24.01 \\
\hline $\mathrm{Li}$ & 5.39 & 1.00 & 5.63 & 5.73 & 5.53 & 5.77 & 5.82 \\
\hline $\mathrm{Mg}$ & 7.65 & 1.00 & 7.57 & 7.59 & 7.35 & 7.71 & 7.64 \\
\hline $\mathrm{N}$ & 14.53 & 0.82 & 14.71 & 14.65 & 14.66 & 13.51 & 14.06 \\
\hline $\mathrm{N}_{2}$ & 15.58 & 0.75 & 16.17 & 16.14 & 16.39 & 14.86 & 15.45 \\
\hline $\mathrm{NH}_{3}$ & 10.82 & 0.78 & 11.17 & 11.15 & 10.58 & 10.34 & 10.72 \\
\hline $\mathrm{Na}$ & 5.14 & 1.00 & 5.22 & 5.35 & 5.11 & 5.50 & 5.50 \\
\hline $\mathrm{NaCl}$ & 9.80 & 0.78 & 9.37 & 9.33 & 9.11 & 8.76 & 9.06 \\
\hline $\mathrm{Ne}$ & 21.56 & 0.76 & 21.55 & 21.54 & 21.06 & 20.54 & 21.10 \\
\hline $\mathrm{O}$ & 13.62 & 0.80 & 13.76 & 13.78 & 13.43 & 13.04 & 13.37 \\
\hline $\mathrm{O}_{2}$ & 12.30 & 0.70 & 13.15 & 13.17 & 13.77 & 11.68 & 12.33 \\
\hline OCS & 11.19 & 0.82 & 11.58 & 11.55 & 11.32 & 10.88 & 11.16 \\
\hline $\mathrm{OH}$ & 13.02 & 0.77 & 13.23 & 13.21 & 12.72 & 12.44 & 12.80 \\
\hline $\mathrm{P}$ & 10.49 & 0.86 & 10.62 & 10.58 & 10.62 & 9.94 & 10.24 \\
\hline $\mathrm{P}_{2}$ & 10.62 & 0.87 & 10.57 & 10.56 & 10.29 & 10.13 & 10.35 \\
\hline $\mathrm{PH}_{3}$ & 10.59 & 0.86 & 10.79 & 10.77 & 10.46 & 10.26 & 10.46 \\
\hline S & 10.36 & 0.85 & 10.56 & 10.55 & 10.38 & 10.12 & 10.31 \\
\hline $\mathrm{S}_{2}$ & 9.55 & 0.76 & 9.86 & 9.87 & 10.17 & 9.05 & 9.42 \\
\hline $\mathrm{SH}_{2}$ & 10.50 & 0.84 & 10.61 & 10.56 & 10.31 & 10.05 & 10.28 \\
\hline $\mathrm{Si}$ & 8.15 & 0.89 & 8.33 & 8.30 & 8.29 & 7.76 & 8.01 \\
\hline $\mathrm{SiH}_{4}$ & 12.30 & 0.84 & 13.15 & 13.17 & 12.94 & 12.31 & 12.70 \\
\hline MAE (eV) & 0.00 & - & 0.33 & 0.32 & 0.36 & 0.52 & 0.25 \\
\hline MAPE (\%) & 0.0 & - & 2.9 & 2.9 & 3.1 & 4.5 & 2.2 \\
\hline
\end{tabular}


TABLE VI. Ionization potentials for selected molecules in the G2 test set for $\operatorname{sc} G W$ and $G_{0} W_{0}$. $\operatorname{sc} G W$ results were obtained for a tier 2 basis set, whereas $G_{0} W_{0} @ \operatorname{PBEh}\left(\alpha^{*}\right)$ results were obtained with a tier 4 basis set augmented with diffuse functions from aug-cc-pV5Z. Experimental reference data were taken from the NIST database. ${ }^{40}$

\begin{tabular}{lccc}
\hline \hline Molecule & Expt. & $\operatorname{sc} G W$ & $G_{0} W_{0} @$ PBEh $\left(\alpha^{*}\right)$ \\
\hline $\mathrm{C}_{2} \mathrm{H}_{2}$ & 11.49 & 10.92 & 11.62 \\
$\mathrm{C}_{2} \mathrm{H}_{4}$ & 10.68 & 10.18 & 10.71 \\
$\mathrm{CH}_{3} \mathrm{Cl}$ & 11.29 & 11.09 & 11.66 \\
$\mathrm{CH}_{4}$ & 13.60 & 14.24 & 14.70 \\
$\mathrm{CO}$ & 14.01 & 13.91 & 14.56 \\
$\mathrm{CO}_{2}$ & 13.78 & 13.70 & 14.18 \\
$\mathrm{Cl}_{2}$ & 11.49 & 11.22 & 11.84 \\
$\mathrm{ClF}$ & 12.77 & 12.52 & 11.50 \\
$\mathrm{FH}$ & 16.12 & 16.22 & 16.29 \\
$\mathrm{~N}_{2}$ & 15.58 & 15.53 & 16.14 \\
$\mathrm{NH}_{3}$ & 10.82 & 10.84 & 11.15 \\
$\mathrm{NaCl}_{2}$ & 8.96 & 9.33 \\
$\mathrm{P}_{2}$ & 9.80 & 9.81 & 10.56 \\
$\mathrm{PH}_{3}$ & 10.62 & 10.33 & 10.77 \\
$\mathrm{SH}_{2}$ & 10.59 & 10.02 & 10.56 \\
$\mathrm{SiH}_{4}$ & 10.50 & 12.71 & 13.17 \\
$\mathrm{MAE}$ & 12.30 & 0.35 & 0.43 \\
$\mathrm{MAPE}$ & 0.00 & 3.1 & 3.4 \\
\hline \hline
\end{tabular}

*atalla@fhi-berlin.mpg.de

${ }^{1}$ S. N. Steinmann, C. Piemontesi, A. Delachat, and C. Corminboeuf, J. Chem. Theor. Comp. 8, 1629 (2012).

${ }^{2}$ G. Sini, J. S. Sears, and J.-L. Bredas, J. Chem. Theor. Comp. 7, 602 (2011).

${ }^{3}$ C. Adamo and V. Barone, J. Chem. Phys. 110, 6158 (1999).

${ }^{4}$ J. P. Perdew, M. Ernzerhof, and K. Burke, J. Chem. Phys. 105, 9982 (1996).

${ }^{5}$ A. D. Becke, J. Chem. Phys. 98, 1372 (1993).

${ }^{6}$ M. A. L. Marques, J. Vidal, M. J. T. Oliveira, L. Reining, and S. Botti, Phys. Rev. B 83, 035119 (2011).

${ }^{7}$ A. Alkauskas, P. Broqvist, and A. Pasquarello, Phys. Status Solidi B 248, 775 (2011).

${ }^{8}$ R. Ramprasad, H. Zhu, P. Rinke, and M. Scheffler, Phys. Rev. Lett. 108, 066404 (2012).

${ }^{9}$ T. Stein, H. Eisenberg, L. Kronik, and R. Baer, Phys. Rev. Lett. 105, 266802 (2010).

${ }^{10}$ S. Refaely-Abramson, R. Baer, and L. Kronik, Phys. Rev. B 84, 075144 (2011).

${ }^{11}$ L. Hedin, Phys. Rev. 139, A796 (1965).

${ }^{12}$ F. Caruso, P. Rinke, X. Ren, M. Scheffler, and A. Rubio, Phys. Rev. B 86, 081102(R) (2012); 88, 075105 (2013).

${ }^{13}$ A. Stan, N. E. Dahlen, and R. van Leeuwen, J. Chem. Phys. 130, 114105 (2009).

${ }^{14}$ C. Rostgaard, K. W. Jacobsen, and K. S. Thygesen, Phys. Rev. B 81, 085103 (2010).

${ }^{15}$ B. Holm and U. von Barth, Phys. Scripta T109, 135 (2004).

${ }^{16}$ P. Rinke, A. Qeish, J. Neugebauer, C. Freysoldt, and M. Scheffler, New J. Phys. 7, 126 (2005).

${ }^{17}$ F. Fuchs, J. Furthmüller, F. Bechstedt, M. Shishkin, and G. Kresse, Phys. Rev. B 76, 115109 (2007).

${ }^{18}$ T. Körzdörfer and N. Marom, Phys. Rev. B 86, 041110 (2012).
${ }^{19}$ N. Marom, F. Caruso, X. Ren, O. T. Hofmann, T. Körzdörfer, J. R. Chelikowsky, A. Rubio, M. Scheffler, and P. Rinke, Phys. Rev. B 86, 245127 (2012).

${ }^{20} \mathrm{~F}$. Bruneval and M. A. L. Marques, J. Chem. Theory Comp. 9, 324 (2013)

${ }^{21}$ J. P. Perdew, K. Burke, and M. Ernzerhof, Phys. Rev. Lett. 77, 3865 (1996).

${ }^{22}$ P. Hohenberg, and W. Kohn, Phys. Rev. 136, B864 (1964).

${ }^{23}$ C. O. Almbladh and U. von Barth, Phys. Rev. B 31, 3231 (1985).

${ }^{24}$ M. Levy, J. P. Perdew, and V. Sahni, Phys. Rev. A 30, 2745 (1984).

${ }^{25}$ P. Mori-Sánchez, A. J. Cohen, and W. Yang, J. Chem. Phys. 125, 201102 (2006).

${ }^{26}$ M. van Schilfgaarde, T. Kotani, and S. Faleev, Phys. Rev. Lett. 96, 226402 (2006).

${ }^{27}$ H. Alves, A. S. Molinari, H. Xie, and A. F. Morpurgo, Nat. Mater. 7, 574 (2008).

${ }^{28}$ I. Avilov, V. Geskin, and J. Cornil, Adv. Func. Mater. 19, 624 (2009).

${ }^{29}$ V. Geskin, R. Stadler, and J. Cornil, Phys. Rev. B 80, 085411 (2009).

${ }^{30}$ V. Blum, R. Gehrke, F. Hanke, P. Havu, V. Havu, X. Ren, K. Reuter, and M. Scheffler, Comp. Phys. Commun. 180, 2175 (2009).

${ }^{31}$ X. Ren, P. Rinke, V. Blum, J. Wieferink, A. Tkatchenko, A. Sanfilippo, K. Reuter, and M. Scheffler, New J. Phys. 14, 053020 (2012).

${ }^{32}$ N. Sai, P. F. Barbara, and K. Leung, Phys. Rev. Lett. 106, 226403 (2011).

${ }^{33}$ A. J. Cohen, P. Mori-Sanchez, and W. Yang, Science 321, 792 (2008).

${ }^{34}$ D. L. Lichtenberger, R. L. Johnston, K. Hinkelmann, T. Suzuki, and F. Wudl, J. Am. Chem. Soc. 112, 3302 (1990). 
${ }^{35}$ I. Ikemoto, K. Samizo, T. Fujikawa, K. Ishii, T. Ohta, and H. Kuroda, Chem. Lett. 3, 785 (1974).

${ }^{36}$ B. Milian, R. Pou-Amerigo, R. Viruela, and E. Orti, Chem. Phys. Lett. 391, 148 (2004).

${ }^{37}$ The choice of this geometry is motivated by a recent experiment in which it was shown that the TTF/TCNQ interface exhibits metallic conduction, ${ }^{27}$ although the individual crystals are large band-gap semiconductors. The mutual crystal interface distance was optimized on the PBE level of theory including van der Waals corrections. ${ }^{47}$ We define the distance between the TTF and the TCNQ cyrstals $d_{0}$ as the distance between the planes spanned by the hydrogen atoms of TTF and the nitrogen atoms of TCNQ (Fig. 2, right), for which we obtain $d_{0}=2.1 \AA$.

${ }^{38}$ T. Körzdörfer, R. M. Parrish, N. Marom, J. S. Sears, C. D. Sherril, and J.-L. Brédas, Phys. Rev. B 86, 205110 (2012).
${ }^{39}$ L. A. Curtiss, P. C. Redfern, K. Raghavachari, and J. A. Pople, J. Chem. Phys. 109, 42 (1998).

${ }^{40} \mathrm{http}: / /$ cccbdb.nist.gov

${ }^{41}$ P. Jurečka, J. Šponer, J. Černý, and P. Hobza, Phys. Chem. Chem. Phys. 8, 1985 (2006).

${ }^{42}$ T. Takatani, E. G. Hohenstein, M. Malagoli, M. S. Marshall, and C. D. Sherrill, J. Chem. Phys. 132, 144104 (2010).

${ }^{43}$ D. Bohm and D. Pines, Phys. Rev. 92, 609 (1953).

${ }^{44}$ X. Ren, P. Rinke, C. Joas, and M. Scheffler, J. Mater. Sci. 47, 7447 (2012).

${ }^{45}$ X. Ren, P. Rinke, G. Scuseria, and M. Scheffler, Phys. Rev. B 88, 035120 (2013).

${ }^{46}$ W. Nelson, P. Bokes, P. Rinke, and R. W. Godby, Phys. Rev. A 75, 032505 (2007).

${ }^{47}$ A. Tkatchenko and M. Scheffler, Phys. Rev. Lett. 102, 073005 (2009). 